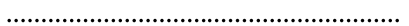

AN. MED INTERNA (Madrid) Vol. 19, N. $^{\circ} 6$, pp. 302-304, 2002

\title{
Fibrilación auricular transitoria inducida por adenosina en una paciente con taquicardia auricular
}

\author{
A. GARCÍA ALBEROLA, A. GÓMEZ AGÜERA, J. J. SÁNCHEZ MUÑOZ, \\ J. MARTÍNEZ SÁNCHEZ, C. LLAMAS LÁZARO, M. VALDÉS CHÁVARRI
}

Servicio de Cardiología. Hospital Universitario Virgen de la Arrixaca. El Palmar. Murcia

ADENOSINE-INDUCED TRANSIENT ATRIAL FIBRILLATION IN A PATIENT WITH ATRIAL TACHYCARDIA

\section{RESUMEN}

La administración de adenosina durante taquicardia auricular suele producir la terminación de la misma o aumentar el grado de bloqueo AV facilitando su diagnóstico. Presentamos un caso de inducción de fibrilación auricular (FA) transitoria por adenosina en una paciente con taquicardia auricular. La transición entre ambos ritmos fue contínua, persistiendo la taquicardia original al finalizar la FA. Este efecto proarrítmico se cree debido al acortamiento de los peridodos refractarios auriculares inducido por la adenosina, disminuyendo la longitud de onda de los circuitos de reentrada y facilitando de este modo la coexistencia simultánea de varios frentes de activación en las aurículas como ocurre en la FA. Aunque es un fenómeno poco común, su reconocimiento es importante para evitar la administración repetida de dosis crecientes de adenosina, que podría llevar a la inducción de FA sostenida.

PALABRAS CLAVE: Adenosina. Taquicardia. Fibrilación auricular.

\begin{abstract}
The administration of adenosine during atrial tachycardia usually terminates the arrhythmia or induces AV block and makes the diagnosis clear. We present a patient with atrial tachycardia in which the adminis tration of adenosine induced a transient atrial fibrillation $(A F)$. A conti nuous transition between both arrhtymias was observed and the original tachycardia persisted after the termination of the AF. This proarrhythmic effect may be due to the adenosinemediated shortening of the atrial refractory periods, which produces a decreased wavelength of the reentry circuits and the potential coexistence of several wavefronts in the atria, favoring the development of $A F$. The recognition of this uncommon effect is important, since the repeated administration of increasing doses of adenosine may induce sustained $A F$.
\end{abstract}

KEY WORDS: Adenosine. Tachycardia. Atrial fibrillation

García Alberola A, Gómez Agüera A, Sánchez Muñoz JJ, Martínez Sánchez J, Llamas Lázaro C, Valdés Chávarri M. Fibrilación auricular transitoria inducida por adenosina en una paciente con taquicardia auricular. An Med Interna (Madrid) 2002; 19: $302-304$.

\section{INTRODUCCIÓN}

La adenosina se considera el fármaco de elección para el tratamiento agudo de las crisis de taquicardia paroxística supraventricular. Administrada en bolo intravenoso presenta un efecto inmediato, una elevada eficacia y un buen perfil de seguridad derivado de la rapidez con que desaparecen sus acciones (1). En pacientes con taquicardia auricular, la administración de adenosina puede terminar la taquicardia o aumentar el grado de bloqueo aurículo-ventricular, lo que pone de manifiesto la actividad auricular en el ECG y facilita el diagnóstico de la arritmia (2). Una tercera posibilidad, mucho más rara, es la inducción de fibrilación auricular (FA) tras la administración del fármaco. Presentamos un caso en el que la administración de $12 \mathrm{mg}$. de adenosina IV a una paciente con taquicardia auricular indujo una FA transitoria de unos segundos de duración con persistencia de la taquicardia auricular al ceder la fibrilación.

\section{CASO APORTADO}

Se trata de una paciente de 48 años que acudió al servicio de urgencias por presentar palpitaciones rápidas. Tenía antecedentes de estenosis pulmonar valvular e infundibular, por lo que se le practicó comisurotomía pulmonar y ampliación del tracto de salida ventricular derecho 14 años antes, encontrándose en buena situación funcional. Refería episodios frecuentes de palpitaciones autolimitadas de carácter paroxístico en los días previos, con buena tolerancia. Consultó por presentar un cuadro de palpitaciones de inicio súbito similar a los descritos, acompañado de naúseas y malestar general. En la exploración destacaba un pulso irregular a 160 I.p.m. con buen estado general y sin signos de insuficiencia cardíaca ni bajo gasto. El ECG a su llegada a urgencias se muestra en la figura 1. Se puede observar una taquicardia irregular de QRS estrecho, con actividad auricular rítmica a unos 190 I.p.m. alternando períodos de conducción 1:1 al ventrículo con otros de bloqueo variable. La frecuencia auricular bajó progresivamente en los minutos siguientes, quedando con una longitud de ciclo estable de unos $420 \mathrm{~ms}$. y manteniendo con este ciclo conducción 1:1 a ventrículo. En ese momento se adminis-

Trabajo aceptado: 13 de diciembre de 2000

Correspondencia: Arcadio García Alberola. Pza. del Roble, 36. 30150 La Alberca (Murcia) 


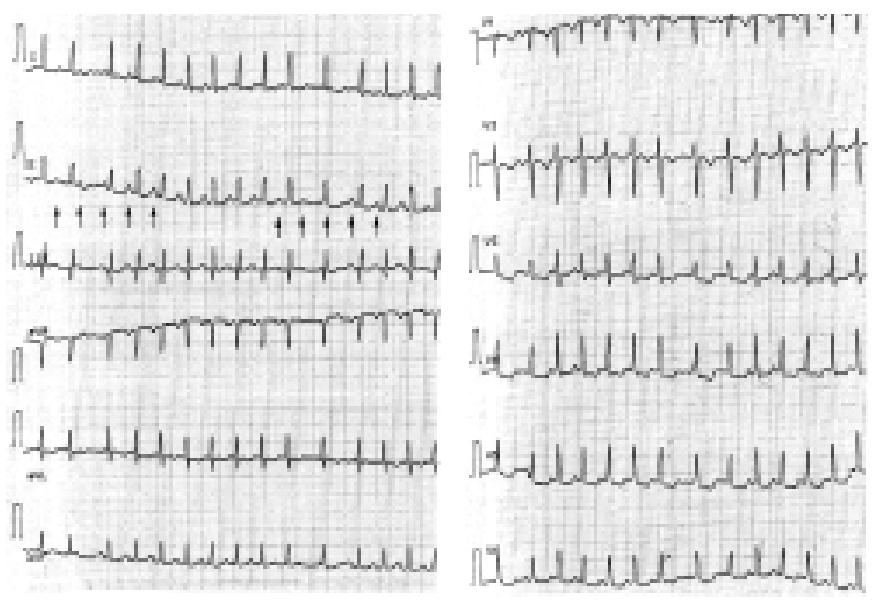

Fig. 1. ECG de 12 derivaciones mostrando la taquicardia auricular con conducción AV variable. En la derivación D2 las flechas señalan ondas $\mathrm{P}^{\prime}$ de activación auricular.

traron $12 \mathrm{mg}$ de adenosina en bolo IV. El efecto del fármaco se presenta en la figura 2. En la tira A, al inicio de la administración, se observa la taquicardia regular descrita. Pocos segundos después el ritmo ventricular se enlentece y se hace irregular, coincidiendo con una actividad auricular más rápida y desorganizada (señalada con puntas de flecha en la figura) propia de la FA. A partir del latido 9 de la tira $\mathrm{C}$ el ritmo se hace de nuevo regular con pequeñas oscilaciones, aparece un complejo ancho y prematuro sugestivo de extrasístole ventricular y el trazado se mantiene regular y estable con una longitud de ciclo similar a la previa a la administración de adenosina. La paciente recibió tratamiento con amiodarona en perfusión y digital, manteniendo taquicardia auricular con frecuencia ventricular controlada y pasando en las horas siguientes a fibrilación auricular estable. Un ecocardiograma mostró importante dilatación del tronco pulmonar y tracto de salida del ventrículo derecho, sin hipertensión pulmonar y con dilatación de ambas aurículas. La paciente rechazó la realización de un estudio electrofisiologico.

\section{DISCUSIÓN}

La inducción de FA es un efecto proarrítmico bien conocido de la adenosina (3-7). Ocurriría entre el 1 y $12 \%$ de los pacientes con taquicardia supraventricular $(6,8)$ y se ha sugerido que podría depender de la dosis y vía de administración (6). La incidencia también parece depender del tipo de taquicardia, siendo en la serie de Glatter (7) del $0 \%$ cuando se administra en taquicardias intranodales y del $15 \%$ en las taquicardias mediadas por vía accesoria. Por el contrario otros autores no encuentran diferencias significativas en este aspecto (6). En pacientes con taquicardia auricular la inducción de FA tras administración de adenosina se ha descrito en 2 de 8 casos en una serie (6) y en el $11 \%$ de 53 pacientes en otra más amplia (7). El mecanismo de inicio de la FA inducida por adenosina ha sido sólo parcialmente analizado. En todos los pacientes descritos por Strickberger (6) la FA se produce después de la terminación de la taquicardia supraventricular por la adenosina, apareciendo el primer extrasístole que inicia la FA sobre ritmo sinusal en el $92 \%$ de los casos. En el único estudio publicado con un número elevado de taquicardias auriculares no se analiza el mecanismo de comienzo de la FA, aunque sí se describe el modo de terminación de la taquicardia auricular y en ningún caso fue debido al desarrollo de fibrila- ción (7). De los datos publicados parece, por tanto, deducirse que la FA aparece generalmente después de haber finalizado la taquicardia supraventricular, por lo que una transición contínua entre ambos ritmos como la representada en la figura 2 sería poco común. Con respecto a la duración de la FA inducida, es muy variable (desde unos segundos hasta más de 20 minutos), pudiendo ser persistente $(5,6,9)$. En el presente caso la duración es de unos 25 sgs. según el trazado de la figura 2. Se han invocado varios mecanismos electrofisiológicos para explicar la inducción de FA por adenosina. Se sabe que este fármaco disminuye la duración del potencial de acción auricular y con ello del período refractario (10), por lo que acortaría la longitud de onda de los circuitos de reentrada auriculares. Este efecto permitiría la presencia simultánea de varios frentes de onda facilitando de este modo la fibrilación $(11,12)$. En nuestro caso la despolarización organizada de las aurículas a partir del foco o circuito de la taquicardia auricular podría haberse fragmentado, generando la activación de varios circuitos independientes y simultáneos como consecuencia del acortamiento de los períodos refractarios por la adenosina. La persistencia de la taquicardia auricular podría producir un efecto parecido como consecuencia del remodelado eléctrico (13), y este hecho a su vez explicaría la degeneración espontánea en FA que se observó después de varias horas. También se han sugerido otros mecanismos en la inducción de FA por adenosina, como la dispersión de períodos refractarios secundaria a la aparición de extrasístoles que generan secuencias de activación con ciclo largocorto (6). El hecho de que la FA se inicie a menudo después de la terminación de la taquicardia supraventricular con adenosina, con el paciente ya en ritmo sinusal y precedida de un extrasístole auricular apoyaría este mecanismo. En nuestro caso, sin embargo, este efecto parece poco relevante, ya que existe una transición directa entre la taquicardia y la fibrilación auricular, sin ciclos largos entre ambos ritmos. Por último, la activación simpática refleja que se produce tras el efecto directo de la adenosina sobre el corazón (14) tampoco parece tener relevancia en el caso descrito, ya que el momento de inicio de la FA coincide con la acción directa de la adenosina sobre el nodo aurículo-ventricular, resultando en una frecuencia ventricular relativamente lenta durante la fibrilación.

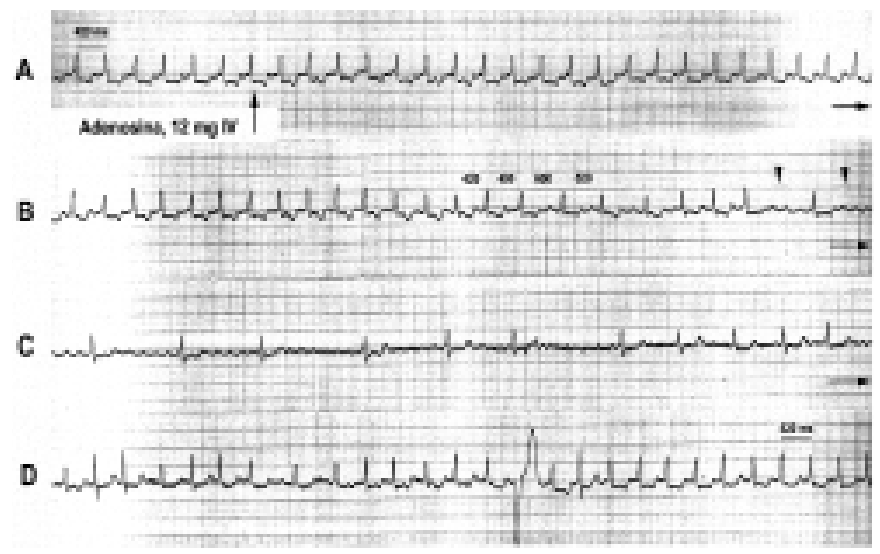

Fig. 2. Inducción de fibrilación auricular transitoria por la administración de adenosina durante taquicardia auricular (registro contínuo). Los números representan las longitudes de ciclo de los RR correspondientes. Las puntas de flecha en la tira B señalan la actividad auricular característica de la fibrilación. 
Implicaciones clínicas: La adenosina se utiliza con fines diagnósticos y terapeúticos en las taquicardias supraventriculares. Desde el punto de vista diagnóstico, la inducción de FA autolimitada con persistencia ulterior de la arritmia previa no debe ser específica de taquicardia auricular, ya que, al menos teóricamente, sería posible tanto en la taquicardia por reentrada intranodal como en las taquicardias mediadas por vías accesorias. En ambos casos, el mecanismo de reentrada que perpetúa la taquicardia finalizaría al iniciarse la FA, pero los últimos frentes de activación de la fibrilación podrían penetrar de forma adecuada en el circuito y reinducir la taquicardia existiendo de esta forma una transición directa entre ambos ritmos como la que se observa en la figura 2. Desde el punto de vista terapeútico es importante reconocer la inducción de FA transitoria y no interpretar la respuesta como simple ausencia de efectividad de la dosis utilizada, evitando la administración repetida de dosis crecientes del fármaco. Teniendo en cuenta que la inducción de FA por adenosina parece bastante reproducible en el mismo paciente (5) y que además puede ser dosisdependiente (6), esta actitud probablemente conduciría al desarrollo de nuevos episodios de FA que podrían ser sostenidos y precisar una cardioversión eléctrica para su terminación $(5,9)$.

\section{Bibliografía}

1. Alzueta J, Cinca J, Coma R, Mont Ll, Rodríguez J, Esteve JJ. Manejo actual de las taquicardias supraventriculares. En: Asín E, Cosín J, De Río A, editores. Normas de actuación clínica en Cardiología. Madrid: Sociedad Española de Cardiología; 1996. p. 240-57.

2. DiMarco JP, Sellers TD, Lerman BB, Greenberg ML, Berne RM, Belardinelli L. Diagnostic and therapeutic use of adenosine in patients with supraventricular tachyarrhythmias. J Am Coll Cardiol 1985; 6: 417-25.

3. Tebbenjohanns J, Pfeiffer D, Schumacher B, Jung W, Manz M, Luderitz $\mathrm{B}$. Intravenous adenosine during atrioventricular reentrant tachycardia: Induction of atrial fibrillation with rapid conduction over an accessory pathway. Pacing Clin Electrophysiol 1995; 18: 743-6.

4. Exner DV, Muzyka T, Gillis AM. Proarrhythmia in patients with the Wolff-Parkinson-White syndrome after standard doses of intravenous adenosine. Ann Intern Med 1995; 122: 351-2.

5. Silverman AJ, Machado C, Baga JJ, Meissner MD, Lehmann MH, Steinman RT. Adenosine-induced atrial fibrillation. Am J Emerg Med 1996; 14: 300-1.

6. Strickberger SA, Man KC, Daoud EG, Goyal R, Brinkman K, Knight BP, Weiss R, Bahu M, Morady F. Adenosine-induced atrial arrhythmia: A prospective analysis. Ann Intern Med 1997; 127: 417-22.

7. Glatter KA, Cheng J, Dorostkar P, Modin G, Talwar S, Al-Nimri M, et al. Electrphysiologic effects of adenosine in patients with supraventricular tachycardia. Circulation 1998; 99: 1034-40.

8. DiMarco JP, Miles W, Akhtar M, Milstein S, Sharma AD, Platia E, et

al. Adenosine for paroxysmal supraventricular tachycardia: dose ranging and comparison with verapamil. Assessment in placebo-controlled, multicenter trials. The Adenosine for PSVT Study Group. Ann Intern Med 1990; 113: 996.

9. Mclntosh-Yellin NL, Drew BJ, Scheinman MM. Safety and efficacy of central intravenous bolus administration of adenosine for termination of supraventricular tachycardia. J Am Coll Cardiol 1993; 22: 741-5.

10. O'Nunain S, Garratt C, Paul V, Debbas N, Ward DE, Camm AJ. Effect of intravenous adenosine on human atrial and ventricular repolarization. Cardiovasc Res 1992; 26: 939-43.

11. Smeets JL, Allessie MA, Lammers WJ, Bonke Fl, Hollen J. The wavelength of the cardiac impulse and reentrant arrhythmias in isolated rabbit atrium. The role of heart rate, autonomic transmitters, temperature and potassium. Circ Res 1986; 58: 96-108

12. Rensma PL. Allessie MA, Lammers WJ, Bonke FI, Schalij H. The length of the excitation wave and susceptibility to reentrant atrial arrhythmias in normal conscious dogs. Circ Res 1 988; 62: 395-410.

13. Wijffels MC, Kirchof CJ, Dorland R, Allessie MA. AF begets AF: A study in awake chronically instrumented goats. Circulation 1995; 92 1954-68.

14. Biaggioni 1, Killian TJ, Mosqueda R, Robertson RM, Robertson D. Adenosine increases sympathetic nerve traffic in humans. Circulation $1991 ; 83: 1668-75$ 